\title{
Small Bowel Imaging in Celiac Disease
}

\author{
Stijn J.B. Van Weyenberg ${ }^{a}$ Chris J.J. Mulder ${ }^{b}$ Jan Hein T.M. Van Waesberghe ${ }^{c}$

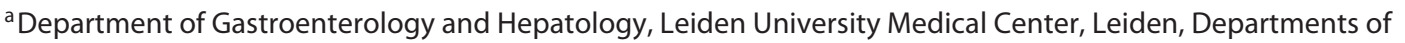 \\ ${ }^{\mathrm{b}}$ Gastroenterology and Hepatology and ${ }^{\mathrm{C}}$ Radiology, VU University Medical Center, Amsterdam, The Netherlands
}

\section{Key Words}

Celiac disease · Radiology · Enteroclysis · Magnetic

resonance imaging $\cdot$ Small intestine

\begin{abstract}
Background: Modern small bowel imaging techniques allow detailed depiction of small-intestinal abnormalities. The role of these techniques in the investigation of celiac disease is increasing, especially in patients with suspected complicated celiac disease. Key Messages: In general, there is no need for radiological small bowel imaging in uncomplicated celiac disease. It is however important that clinicians and radiologists are aware of certain specific radiological findings that may suggest celiac disease, especially since celiac disease is often not considered in adult patients, and small bowel radiology may be performed before specific tests for celiac disease. Radiological abnormalities can be observed with both conventional small bowel radiology studies, like small bowel follow-through or double-contrast small bowel enteroclysis, and newer modalities, like computed tomography or magnetic resonance enterography or enteroclysis. These signs include a decreased number of jejunal folds, an increased number of ileal folds, small bowel dilatation, wall thickening and intussusception. Extraintestinal abnormali-
\end{abstract}

ties include mesenteric lymphadenopathy, vascular changes and splenic atrophy. Abnormalities congruent with refractory celiac disease type II include a severe decrease in jejunal folds, infiltration of the mesenteric fat and thickening of the small bowel wall. Additionally, a severely decreased splenic volume may indicate complicated celiac disease. Malignant complications of celiac disease, such as enteropathy-associated T-cell lymphoma and small-intestinal adenocarcinoma, can be reliably investigated with cross-sectional enteroclysis techniques. Conclusions: Small bowel imaging and especially cross-sectional enteroclysis techniques are important extensions to the diagnostic workup of clinicians involved in the care of patients with celiac disease, especially those with suspected complicated disease.

(c) 2015 S. Karger AG, Basel

\section{Introduction}

With the current possibilities of serology and histology, radiology plays no important role in the diagnosis of celiac disease (CD). However, it is important that radiological features associated with $\mathrm{CD}$ are recognized, especially since in patients with nonspecific symptoms abdominal imaging may be performed before the diagnosis

\section{KARGER 125}

(c) 2015 S. Karger AG, Base

0257-2753/15/0332-0252\$39.50/0

E-Mail karger@karger.com

www.karger.com/ddi
Stijn J.B. Van Weyenberg

Department of Gastroenterology and Hepatology

Leiden University Medical Center

Albinusdreef 2, NL-2333 ZA Leiden (The Netherlands)

E-Mail s.j.b.van_weyenberg@lumc.nl 
Table 1. Diagnostic modalities in small bowel radiology

\begin{tabular}{|c|c|c|c|c|c|c|c|c|}
\hline Modality & Invasiveness & Enteral contrast & $\begin{array}{l}\text { Radiation } \\
\text { exposure }\end{array}$ & $\begin{array}{l}\text { Luminal } \\
\text { detail }\end{array}$ & $\begin{array}{l}\text { Wall } \\
\text { detail }\end{array}$ & $\begin{array}{l}\text { Extraintestinal } \\
\text { detail }\end{array}$ & Availability & Costs \\
\hline Plain abdominal X-ray & - & None & + & + & - & - & ++++ & + \\
\hline SBFT & + & Oral & +++ & ++ & + & - & +++ & ++ \\
\hline Conventional enteroclysis & +++ & Nasojejunal tube & +++ & +++ & + & - & ++ & ++ \\
\hline Double-contrast enteroclysis & +++ & Nasojejunal tube & +++ & ++++ & ++ & - & + & ++ \\
\hline Abdominal ultrasound & - & None & - & + & + & ++ & ++++ & + \\
\hline CT enterography & ++ & Oral & +++ & +++ & +++ & +++ & +++ & ++ \\
\hline CT enteroclysis & +++ & Nasojejunal tube & +++ & ++++ & ++++ & +++ & ++ & ++ \\
\hline MR enterography & ++ & Oral & - & +++ & +++ & +++ & ++ & +++ \\
\hline MR enteroclysis & +++ & Nasojejunal tube & + (tube placement) & ++++ & ++++ & +++ & + & +++ \\
\hline
\end{tabular}

of $\mathrm{CD}$ is considered and specific testing has been performed. In patients with nonresponsive $\mathrm{CD}$, the possibility of refractory $\mathrm{CD}(\mathrm{RCD})$, small bowel adenocarcinoma or lymphoma has to be considered. In these circumstances, small bowel imaging may provide important information.

This review aims to provide clinicians involved with the care for patients with CD with some basic knowledge on the strengths and limitations of modern small bowel radiology. Additionally, it aims to inform on the diagnostic accuracy of radiological findings associated with $\mathrm{CD}$, as well as its complications, including RCD and small bowel malignancies.

\section{Modalities in Small Bowel Radiology}

In order to be able to appreciate the findings of small bowel radiology, or to efficiently order small bowel radiology, some knowledge on the possibilities and limitations of the different modalities and ways of contrast delivery is important (table 1). The ideal small bowel imaging method is noninvasive, does not require potentially toxic (intravenous) contrast agents, does not use ionizing radiation, and is able to depict the complete small-intestinal lumen, bowel wall, as well as extraluminal structures. In addition, the ideal modality needs to be widely available, result in easy to interpret images and be costeffective [1]. Small bowel radiological modalities can be classified by the way the image is acquired: fluoroscopy, ultrasound (US), computed tomography (CT) or magnetic resonance (MR) imaging. Except for the modality used, another important factor is whether or not luminal contrast agents are being used, and if so, how these are administered: orally (enterography) or by means of a nasojejunal catheter (enteroclysis). The latter is considered to be more invasive, but allows periprocedural increasing of luminal contrast dose when suboptimal bowel distention is seen, which seems to be particularly important when information on the jejunum is needed [2].

Up to 10 years ago, most small bowel radiology was performed using fluoroscopic techniques. The most prevalent technique was small bowel follow-through (SBFT), which requires the ingestion of at least 0.5 liters of barium suspension. Fluoroscopy is performed when the barium progresses through the intestine. Manual palpation is usually needed to separate individual small bowel loops. Although easy to perform, the poor distention of the small bowel as well as the poor separation of segments limit its accuracy [1]. These problems can be overcome when the barium is administered directly into the small intestine using a fluoroscopically placed nasojejunal catheter, a technique that is called enteroclysis. Even better distention can be achieved when this is followed by administration of methylcellulose suspension or air to enable optimal distension of individual small bowel loops [3]. Limitations of fluoroscopic techniques include the limited information they provide on mural and extraintestinal abnormalities as well as the need for ionizing radiation.

CT is a widely available cross-sectional imaging method, often used in the evaluation of abdominal symptoms. In routine settings, too little luminal contrast agent is administered to prevent collapse of the small-intestinal lumen, and therefore information on the small intestine in limited. Bowel distention can be achieved either by increasing the dose of orally ingested contrast medium (enterography) or by administering methylcellulose (neutral enteral contrast) or barium (positive enteral contrast) directly into the small bowel by means of a nasojejunal catheter (enteroclysis) [1]. 
1

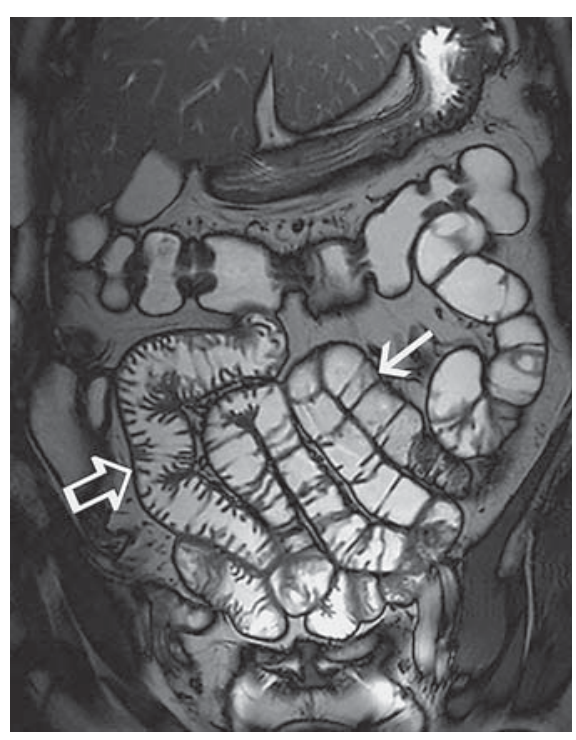

2

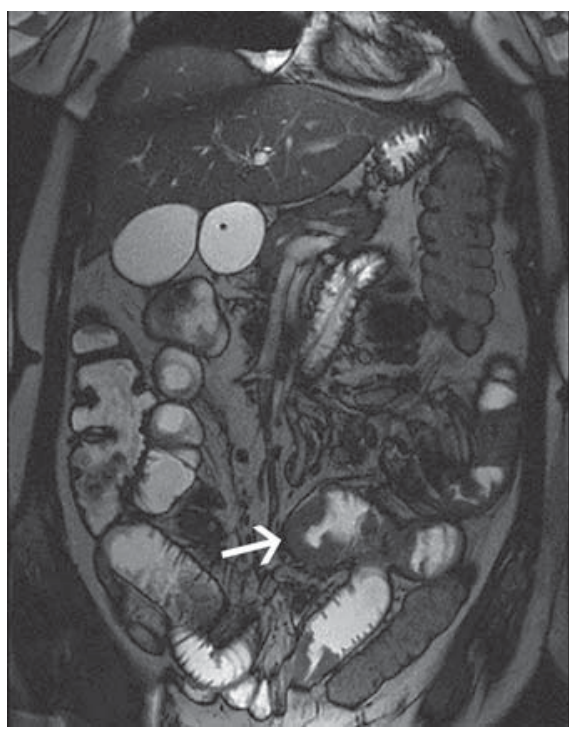

Fig. 1. Coronal MR enteroclysis image shows JFPR in a 45-year-old female with RCD type I. The number of jejunal folds per $5 \mathrm{~cm}$ is 2 (arrow), whereas the number of ileal folds per $5 \mathrm{~cm}$ is 7 (open arrow).

Fig. 2. Coronal MR enteroclysis image shows EATL (arrow) in a patient with RCD type II.
With the development of fast imaging sequences, small bowel imaging by MR has become possible as well. Advantages include excellent soft tissue contrast and the lack of ionizing radiation. The latter allows for repeated dynamic imaging, so multiple series can be obtained during the same study. This is especially helpful to follow the progression of bowel distention during MR enteroclysis, and allows discrimination between intermittent spasms and stenosis, which often appear the same on static imaging [4]. As in CT, enteroclysis is considered to be more reliable than enterography, although more invasive.

Abdominal US is an easy to perform, noninvasive and readily available imaging modality. However, the lack of bowel preparation often results in intestinal gas or collapse of the bowel wall hindering detailed imaging of the small bowel. However, abdominal US provides better functional evaluation than static small bowel imaging modalities, and especially in case of small bowel obstruction when loops are filled with fluid, the detail with which the intestine can be depicted is impressive [1].

\section{Radiology in Uncomplicated CD}

Diagnosis of CD in adults is by serology and duodenal biopsy while the patient is on a gluten-containing diet [5]. The performance of currently available methods for serological diagnosis of CD is very good [6]. For instance, the sensitivity and specificity of IgA anti-endomysial antibodies have been reported to be around 0.84 and 1.00, respectively. For IgA tissue transglutaminase (tTG) antibodies, the sensitivity and specificity have been reported to be around 0.93 and 0.95 , respectively [6]. When combined, the diagnostic accuracy of serological tests may approximate 1.0. Despite these performance characteristics, it is generally advised to perform duodenal biopsies, especially in adult patients with gastrointestinal symptoms, before a diagnosis of CD is established [5]. Diagnostic accuracy of duodenal (and bulbar) biopsy specimens for the diagnosis of $\mathrm{CD}$ is high, especially when over 4 specimens are obtained [7]. However, one has to be aware that villous atrophy can be caused by more conditions than CD alone, which is why the combination of serology and histology is advocated. In children, the diagnostic accuracy of tTG antibodies is considered that good that in case of tTG raised more than $10 \times$ the upper limit of normal, confirmatory duodenal biopsies are no longer required in order to avoid invasive endoscopy [8].

From the above, it can be concluded that modern diagnostic tests for CD are that good that there is no need for radiological methods to investigate the suspected presence of $\mathrm{CD}$ in children or adults. However, serological tests for $\mathrm{CD}$, and to a lesser extent duodenal biopsies, are disease-specific tests. In other words, these tests are usually only ordered when a distinct suspicion of CD has arisen. However, the heterogeneous clinical picture of CD remains a challenge to many physicians, and often results in a diagnostic delay of up to 12 years, as well as excessive use of health services [9]. Although there is increasing awareness of $\mathrm{CD}$, this may not be the case for $\mathrm{CD}$ present- 
Table 2. Radiological findings in CD and its complications

\begin{tabular}{lll}
\hline Condition & Intestinal findings & Extraintestinal findings \\
\hline Untreated CD & $\begin{array}{l}\text { Decreased number of jejunal folds } \\
\text { Increased number of ileal folds }\end{array}$ & $\begin{array}{l}\text { Presence of nonenlarged mesenteric lymph nodes } \\
\text { Presence of enlarged mesenteric lymph nodes } \\
\text { JFPR } \\
\text { Jejunal dilatation } \\
\text { Wall thickening } \\
\text { Intussusception }\end{array}$ \\
& As in untreated CD & \\
\hline RCD type I & Ulcers & As in untreated CD \\
\hline RCD type II & Strictures & Mesenteric fat infiltration \\
& Decreased number of jejunal folds & Cavitated lymph nodes \\
& Diffuse bowel wall thickening & Severely decreased splenic volume \\
& Long, smooth small bowel mass & Presence of enlarged mesenteric lymph nodes \\
\hline EATL & Focal small bowel wall thickening & \\
\hline Small bowel adenocarcinoma & Circular apple core-like lesion & Presence of enlarged mesenteric lymph nodes \\
& Focal wall thickening & \\
& Prestenotic dilatation & \\
\hline
\end{tabular}

ing with nonspecific, or nongastrointestinal symptoms, which is especially cumbersome now that the classical presenting symptom of CD is less prevalent [10]. For instance, a recent survey among practicing hematologists in the United States revealed that only $8.6 \%$ believed that patients with iron deficiency anemia should be screened for CD [11]. In patients with anemia, unintentional weight loss, liver test abnormalities or abdominal pain, it is not unlikely that radiological imaging is performed before $\mathrm{CD}$ is considered an option. Radiological imaging has quite often been performed in the diagnostic process of symptoms that were not recognized as being part of the spectrum of CD. This may especially be true in older individuals, where radiological imaging is often used to exclude malignancy. Therefore, it is important that clinicians are aware of the spectrum of radiological findings that may indicate the presence of CD (table 2).

The most striking radiological abnormalities encountered in $\mathrm{CD}$ are abnormalities of the intestinal fold pattern (fig. 1). The normal jejunum has over 10 folds per 5 $\mathrm{cm}$, whereas the ileum usually has less than 4 folds per 5 $\mathrm{cm}$ [12]. In CD, the number of jejunal folds is often decreased. In severe cases, complete loss of jejunal folds can result in an appearance not unlike that of the colon ('moulage sign') [13]. In contrast, the number of ileal folds is often increased in CD. This probably is the result of ileal adaptation in order to compensate for the loss of absorptive capacity of the jejunum. When the number of ileal folds per $5 \mathrm{~cm}$ exceeds the number of jejunal folds per 5 $\mathrm{cm}$, this is called jejunoileal fold pattern reversal (JFPR). This finding, which was first described by Bova in 1985, is considered to be the most specific radiological finding in CD [14]. Lomoschitz et al. [15] identified JFPR on conventional enteroclysis studies in 16/27 patients with CD, compared to $0 / 123$ control patients. Using CT enteroclysis, Soyer et al. [12] found the median number of jejunal and ileal folds in patients with CD was 7 and 8 per $5 \mathrm{~cm}$, respectively, compared to 10 and 4 per $5 \mathrm{~cm}$ in controls. The sensitivity and specificity of JFPR in the diagnosis of $\mathrm{CD}$ was 0.64 and 1.00 , respectively, with an overall accuracy of 0.82 . Adequate distention of the small intestine is however mandatory. In a study on abdominal CT after oral administration of $800-1,000 \mathrm{ml}$ Gastrografin, it was not possible to count the number of jejunal folds in $7 / 52$ (13.5\%) study subjects [16]. Again, JFPR was only found in patients with CD. Despite the lack of special bowel preparation, Bartusek et al. [17] were able to diagnose a decreased number of jejunal folds in $90 \%$, and an increase in ileal folds in $75 \%$ of patients with CD using abdominal US. Abnormalities in fold pattern usually resolve after the introduction of a gluten-free diet [18].

Measurements of small bowel wall thickness usually require cross-sectional imaging and optimal distention of the small intestine in order to prevent false-positive findings caused by collapsed loops. In optimally distended loops, the small bowel wall is usually $<3-4 \mathrm{~mm}$ thick. 
With CT enterography, Tomei et al. [19] found this feature in $21 \%$ of patients with CD, the mean thickness being $9 \mathrm{~mm}$ (range 5-18). In a study on CT enteroclysis that included 44 patients with $\mathrm{CD}$ and 44 control subjects, the sensitivity and specificity of diffuse jejunal wall thickening were 0.66 and 0.98 [12]. Establishing bowel wall thickness with fluoroscopy is less reliable.

Small bowel hypotonia associated with CD may result in jejunal dilatation. In normal subjects, the diameter of the jejunum is usually $<4 \mathrm{~cm}$, although this depends on the way it is studied. Using SBFT, McCrae and Sweet [20] showed that in children with $\mathrm{CD}$, the diameter of the jejunum was increased when compared to controls. It could be that the process of enteroclysis diminishes the value of jejunal diameter as a relevant feature. Lomoschitz et al. [15] identified jejunal dilatation in 5/27 patients with $C D$, and in 9/123 control subjects using conventional enteroclysis. Soyer et al. [12] in a study on CT enteroclysis found the sensitivity and specificity of jejunal dilatation to be 0.16 and 0.95 , respectively. In older days, the dilatation of the jejunum as well as the excess fluid in the jejunum resulted in the uneven distribution of barium throughout the jejunum. This finding, called flocculation, has been reported in up to $66 \%$ of patients with $\mathrm{CD}$ undergoing SBFT, but was much less prevalent with enteroclysis techniques $[15,21]$. With modern barium solutions, flocculation is now very rare.

Intussusception is a finding in which one part of the intestine slides into the adjacent part. It is important to realize that intussusceptions in $\mathrm{CD}$ are usually transient and may be completely asymptomatic. Intussusception is the most common cause of small bowel obstruction in children aged 3 months to 5 years [22]. Usually, no lead point can be identified. A study from the United States showed that $1.2 \%$ of children with CD had experienced a symptomatic intussusception $<9$ months before the diagnosis of $\mathrm{CD}$. In a large control population, this had occurred in $0.07 \%$ of children, within the same time frame [23]. On SBFT, intussusception results in a spring coillike configuration. On US and cross-sectional imaging, a target sign, reflecting the bowel-in-bowel, is pathognomonic [13]. In adults with CD, intussusception has been reported in up to $20 \%$ of patients examined with SBFT [24]. Using abdominal US, Bartusek et al. [17] found transient intussusception in $30 \%$ of patients with $\mathrm{CD}$, which reflects the dynamic nature of US.

Extraintestinal findings in $\mathrm{CD}$ include changes in blood vessels, lymph nodes and splenic atrophy. All these findings can only be reliably depicted in cross-sectional imaging and to a lesser extent with abdominal US.
Changes in blood flow in CD result in several findings. Abdominal US studies have shown that basal mesenteric blood flow is increased in patients with $\mathrm{CD}$, and that the caliber of the superior mesenteric artery is increased [25]. On cross-sectional imaging, increased blood flow results in a specific image often referred to as vascular engorgement, which is defined as an increase in size and number of proximal and mesenteric vessels, as well as an increase in the number of distal mesenteric vessels adjacent to the small bowel loops [12]. This finding has a reported sensitivity and specificity of 0.64 and 0.91 , respectively [12]. This increased blood flow can also be observed in the bowel wall itself. Masselli et al. [26] found that in CD dynamic contrast-enhanced MR imaging showed increased enhancement of the duodenal wall after administration of intravenous contrast. Additionally, this enhancement was very fast, as was the washout of contrast, which probably indicates increased capillary permeability associated with chronic inflammation. As is clear, such measurements require repeated imaging, which is one of the main benefits of MR imaging, compared to CT.

The presence of nonenlarged lymph nodes frequently occurs in the general population, although it is more frequently encountered in patients with CD. Using abdominal US, Bartusek et al. [17] found mesenteric lymph nodes in $95 \%$ of patients with CD. Enlarged mesenteric lymph nodes, defined as measuring $>1 \mathrm{~cm}$ in their shortaxis length, were found in $18 \%$ of patients with CD [12]. Care is needed in evaluating the aspect of enlarged lymph nodes: enlarged lymph nodes with central hypoattenuation (CD) or a hypoechoic core (abdominal US) are highly suggestive of cavitating lymph node syndrome, especially when associated with splenic atrophy. This very rare syndrome may indicate the presence of lymphoma. Although mortality rates of up to $50 \%$ have been reported, spontaneous recovery has been reported as well [13]. Hyposplenism is associated with $\mathrm{CD}$, although the exact mechanism is unclear. On imaging studies, splenic atrophy is often encountered. With CT, Soyer et al. [12] found the median splenic volume of controls was $254 \mathrm{~cm}^{3} \mathrm{com}$ pared to $157 \mathrm{~cm}^{3}$ in patients with untreated CD. Using receiver operating characteristics curve analysis, splenic atrophy was defined as the presence of a splenic volume $<145 \mathrm{~cm}^{3}$. Sensitivity and specificity of this finding were 0.47 and 0.98 , respectively.

One has to be aware that many signs associated with $\mathrm{CD}$ can also be observed in other conditions resulting in malabsorption. The reported sensitivities and specificities of signs may be inflated by the much larger prevalence of $\mathrm{CD}$ than other conditions, like auto-immune enter- 
opathy and Whipple's disease. In many more rare or emerging small-intestinal conditions, no studies on radiological findings have been performed. Therefore, radiological findings that may indicate $\mathrm{CD}$ need serological and histological confirmation before a diagnosis of $\mathrm{CD}$ can be established.

\section{Radiology in Complicated CD}

The role of radiology in patients with complicated CD is much more important than it is in patients with uncomplicated $\mathrm{CD}$. In patients with nonresponsive $\mathrm{CD}$, symptoms may be caused by RCD, or small-intestinal malignancies like small bowel adenocarcinoma or enteropathy-associated T-cell lymphoma (EATL). In these patients, abnormalities are often not within reach of conventional gastroscopes, and especially in the presence of symptoms that may indicate small bowel obstruction, video capsule endoscopy is contraindicated [27, 28]. Therefore, small bowel radiology can be a helpful tool to investigate these patients (fig. 2). However, little is known on radiological findings in patients with complicated CD, and unfortunately, most recent studies were all performed in one expert center, limiting their generalizability.

Ulcerative jejunitis may appear on double-contrast enteroclysis studies as thickened segments with ulcerative lesions and stenosis [13]. On cross-sectional imaging, the bowel wall thickening is the most striking abnormality. Discrimination from lymphoma is often not possible. However, there are no studies that have investigated the diagnostic accuracy of these findings.

Mallant et al. [29] reported on CT enterography performed in patients with CD because of persisting abdominal symptoms and/or suspicion of EATL. The final diagnosis was uncomplicated CD in 14, RCD type I in 10, RCD type II in 15 and EATL in 7. For analysis, patients with uncomplicated CD or RCD type II were put in group $\mathrm{A}$, and patients with RCD II and EATL in group $\mathrm{B}$. There were no statistical differences regarding fold patterns. However, because of lack of intraluminal contrast or lack of distention of small bowel loops, the number of jejunal folds could not be counted in $43 \%$ of all patients. Jejunal dilation or increased wall thickness did not differ significantly between both groups. Intussusception was found in 1 patient in group $\mathrm{A}$, and in 5 patients in group B, although this difference failed to reach statistical significance. The presence of mesenteric lymph nodes was similar in both groups, but the presence of enlarged lymph nodes was only found in 5 patients in group B. Splenic volume $<122 \mathrm{~cm}^{3}$ was observed in $27 \%$ of patients in group $\mathrm{A}$, and $73 \%$ of patients in group $\mathrm{B}$, a difference that was statistically significant.

Researchers from the same group also studied MR enteroclysis in patients with nonresponsive CD [30]. Patients with de-novo EATL were not included in this study. Radiological parameters were evaluated in a test group consisting of 28 patients (uncomplicated $\mathrm{CD}, \mathrm{n}=$ 10; RCD type I, $\mathrm{n}=8$; RCD type II, $\mathrm{n}=10$ ). No differences between patients with uncomplicated CD or RCD type I could be observed. Multivariate analysis identified three parameters associated with the presence of RCD type II: the presence of $<10$ jejunal folds per $5 \mathrm{~cm}$, diffuse thickening of the small bowel wall, and infiltration of the mesenteric fat. The median splenic volume in patients with RCD type II was $117 \mathrm{~cm}^{3}$ compared to $212 \mathrm{~cm}^{3}$ in patients without RCD II. Using the optimal cutoff value of $<160 \mathrm{~cm}^{3}$, splenic atrophy was present in $33 \%$ of patients without RCD type II, and 70\% of patients with RCD type II, although this difference was not statistically significant in multivariate analysis. The three identified features associated with RCD II were tested in a validation group consisting of 40 patients, 15 of whom had RCD type II. For the presence of $<10$ jejunal folds per $5 \mathrm{~cm}$, sensitivity and specificity were 0.93 and 0.88 , respectively. For mesenteric fat infiltration, this was 0.87 and 0.80 , and for diffuse bowel wall thickening, this was 0.47 and 0.84 . A positive MR score was defined as 2 or more of these three features present, and showed sensitivity and specificity of 0.87 and 0.96 , respectively, for the diagnosis of RCD type II. This MR score was also associated with mortality: 5 -year survival was $95 \%$ in patients with a negative MR score, and $56 \%$ in patients with a positive MR score. In 8 patients, a small bowel malignancy was present, which was detected by MR enteroclysis in 7 , resulting in a specificity and sensitivity of 0.88 and 0.97 , respectively, for the diagnosis of small bowel malignancy in patients with CD.

The Amsterdam group also compared findings of CT enterography and ${ }^{18} \mathrm{~F}$-FDG-PET performed in 8 patients with EATL and 30 patients with RCD type II [31]. ${ }^{18} \mathrm{~F}$ FDG-PET could reveal sites histologically proven to be EATL in all 8 patients, whereas CT showed normal findings in 1 patient with EATL. ${ }^{18}$ F-FDG-PET detected unsuspected extraintestinal sites affected by EATL in 2 patients. CT showed abnormalities such as a thickened small bowel wall or lymphadenopathy in 14 patients with RCD lacking evidence of EATL at follow-up. ${ }^{18} \mathrm{~F}-\mathrm{FDG}$ PET findings were positive in 3 and equivocal in another 3 patients with RCD. ${ }^{18} \mathrm{~F}$-FDG-PET was more sensitive 
and specific than CT ( 100 vs. $87 \%$ and 90 vs. $53 \%$, respectively).

Lohan et al. [32] found that lymphomas in patients with CD were more often solitary, over $10 \mathrm{~cm}$ in length and appearing smoother than lymphomas in patients who did not have CD.

Small bowel adenocarcinomas in CD are often, but not solely, located in the proximal jejunum. On SBFT and in conventional enteroclysis studies, they appear as apple core lesions. On cross-sectional imaging, they usually show as solitary, mass-forming lesions, with a predominant intraluminal growth pattern [33, 34].

Besides the studies mentioned, there is limited additional information on the reliability of small bowel imaging for detecting cancer in patients with $\mathrm{CD}$. However, there is emerging evidence that in the detection of small bowel neoplasms in other patient groups, cross-sectional enteroclysis studies are the preferred method, and may even perform better than video capsule endoscopy $[2,33$, 35]. Enterography studies often do not result in adequate distention of the jejunum, which is essential in the diagnosis of intraluminal abnormalities.

\section{Conclusions}

Small bowel radiology may reveal striking abnormalities in patients with uncomplicated CD. However, in light of the excellent performance characteristics of CD serology and small bowel histology, its role in the diagnosis is limited. However, radiological findings may be the first clues that point physicians to the diagnosis of $\mathrm{CD}$.

The role of radiology in complicated CD seems to be more important, especially when patients present with symptoms that may indicate RCD type II or small bowel malignancy. However, more studies from more centers are needed, which should include a comparison with capsule endoscopy and flexible enteroscopy. Future research should also investigate combined modalities such as PET$\mathrm{CT}$ and PET-MR imaging.

\section{Disclosure Statement}

All authors declare that they have no competing interests.

\section{References}

1 Van Weyenberg SJ, Van Waesberghe JH, Ell C, Pohl J: Enteroscopy and its relationship to radiological small bowel imaging. Gastrointest Endosc Clin N Am 2009;19:389-407.

2 Masselli G, Gualdi G: MR imaging of the small bowel. Radiology 2012;264:333-348.

3 Maglinte DD, Kohli MD, Romano S, Lappas JC: Air (CO2) double-contrast barium enteroclysis. Radiology 2009;252:633-641.

4 Masselli G, Gualdi G: CT and MR enterography in evaluating small bowel diseases: when to use which modality? Abdom Imag 2013;38: 249-259.

5 Ludvigsson JF, Bai JC, Biagi F, Card TR, Ciacci C, Ciclitira PJ, et al: Diagnosis and management of adult coeliac disease: guidelines from the British Society of Gastroenterology. Gut 2014;63:1210-1228.

6 Schyum AC, Rumessen JJ: Serological testing for celiac disease in adults. United European Gastroenterol J 2013;1:319-325.

7 Rubio-Tapia A, Hill ID, Kelly CP, Calderwood AH, Murray JA, American College of G: ACG clinical guidelines: diagnosis and management of celiac disease. Am J Gastroenterol 2013; 108:656-676; quiz 77 .

8 Murch S, Jenkins H, Auth M, Bremner R, Butt A, France S, et al: Joint BSPGHAN and Coeliac UK guidelines for the diagnosis and management of coeliac disease in children. Arch Dis Childhood 2013;98:806-811.
9 Fuchs V, Kurppa K, Huhtala H, Collin P, Maki M, Kaukinen K: Factors associated with long diagnostic delay in celiac disease. Scand J Gastroenterol 2014:1-7.

10 Rampertab SD, Pooran N, Brar P, Singh P, Green PH: Trends in the presentation of celiac disease. Am J Med 2006;119:355 e9-e14.

11 Smukalla S, Lebwohl B, Mears JG, Leslie LA, Green PH: How often do hematologists consider celiac disease in iron-deficiency anemia? Results of a national survey. Clin Adv Hematol Oncol 2014;12:100-105.

12 Soyer P, Boudiaf M, Dray X, Fargeaudou Y, Vahedi K, Aout M, et al: CT enteroclysis features of uncomplicated celiac disease: retrospective analysis of 44 patients. Radiology 2009;253:416-424.

13 Buckley O, Brien JO, Ward E, Doody O, Govender $\mathrm{P}$, Torreggiani WC: The imaging of coeliac disease and its complications. Eur J Radiol 2008;65:483-490.

14 Bova JG, Friedman AC, Weser E, Hopens TA, Wytock DH: Adaptation of the ileum in nontropical sprue: reversal of the jejunoileal fold pattern. Am J Roentgenol 1985;144:299-302.

15 Lomoschitz F, Schima W, Schober E, Turetschek K, Kaider A, Vogelsang H: Enteroclysis in adult celiac disease: diagnostic value of specific radiographic features. Eur Radiol 2003;13:890-896.
16 Tomei E, Marini M, Messineo D, Di Giovambattista F, Greco M, Passariello R, et al: Computed tomography of the small bowel in adult celiac disease: the jejunoileal fold pattern reversal. Eur Radiol 2000;10:119-122.

17 Bartusek D, Valek V, Husty J, Uteseny J: Small bowel ultrasound in patients with celiac disease. Retrospective study. Eur J Radiol 2007; 63:302-306.

18 van den Bosch HC, Tjon a Tham RT, Gooszen AW, Fauquenot-Nollen JM, Lamers CB: Celiac disease: small-bowel enteroclysis findings in adult patients treated with a gluten-free diet. Radiology 1996;201:803-808.

19 Tomei E, Diacinti D, Marini M, Mastropasqua M, Di Tola M, Sabbatella L, et al: Abdominal CT findings may suggest coeliac disease. Dig Liver Dis 2005;37:402-406.

20 McCrae WM, Sweet EM: Radiology in diagnosis of coeliac disease. BMJ 1964;2:163-164.

21 Herlinger H, Maglinte DD: Jejunal fold separation in adult celiac disease: relevance of enteroclysis. Radiology 1986;158:605-611.

22 Lehnert T, Sorge I, Till H, Rolle U: Intussusception in children - clinical presentation, diagnosis and management. Int J Colorect Dis 2009;24:1187-1192.

23 Reilly NR, Aguilar KM, Green PH: Should intussusception in children prompt screening for celiac disease? J Pediatr Gastroenterol Nutr 2013;56:56-59. 
24 Cohen MD, Lintott DJ: Transient small bowel intussusception in adult coeliac disease. Clin Radiol 1978;29:529-534.

25 Rettenbacher T, Hollerweger A, Macheiner P, Huber S, Gritzmann N: Adult celiac disease: US signs. Radiology 1999;211:389-394.

26 Masselli G, Picarelli A, Di Tola M, Libanori V, Donato G, Polettini E, et al: Celiac disease: evaluation with dynamic contrast-enhanced MR imaging. Radiology 2010;256:783-790.

27 Van Weyenberg SJ, Smits F, Jacobs MA, Van Turenhout ST, Mulder CJ: Video capsule endoscopy in patients with nonresponsive celiac disease. J Clin Gastroenterol 2013;47:393399.

28 Van Weyenberg SJ, Van Turenhout ST, Bouma G, Van Waesberghe JH, Van der Peet DL, Mulder CJ, et al: Double-balloon endoscopy as the primary method for small-bowel video capsule endoscope retrieval. Gastrointest Endosc 2010;71:535-541.
29 Mallant M, Hadithi M, Al-Toma AB, Kater M, Jacobs M, Manoliu R, et al: Abdominal computed tomography in refractory coeliac disease and enteropathy associated T-cell lymphoma. World J Gastroenterol 2007;13:16961700.

30 Van Weyenberg SJ, Meijerink MR, Jacobs MA, van Kuijk C, Mulder CJ, van Waesberghe $\mathrm{JH}: \mathrm{MR}$ enteroclysis in refractory celiac disease: proposal and validation of a severity scoring system. Radiology 2011;259:151-161.

31 Hadithi M, Mallant M, Oudejans J, van Waesberghe JH, Mulder CJ, Comans EF: 18F-FDG PET versus $\mathrm{CT}$ for the detection of enteropathy-associated T-cell lymphoma in refractory celiac disease. J Nucl Med 2006;47:16221627.
32 Lohan DG, Alhajeri AN, Cronin CG, Roche CJ, Murphy JM: MR enterography of smallbowel lymphoma: potential for suggestion of histologic subtype and the presence of underlying celiac disease. Am J Roentgenol 2008; 190:287-293.

33 Van Weyenberg SJ, Meijerink MR, Jacobs MA, Van der Peet DL, Van Kuijk C, Mulder $\mathrm{CJ}$, et al: MR enteroclysis in the diagnosis of small-bowel neoplasms. Radiology 2010;254: 765-773.

34 Masselli G, Gualdi G: Evaluation of small bowel tumors: MR enteroclysis. Abdom Imaging 2010;35:23-30.

35 Van Weyenberg SJ, Bouman K, Jacobs MA, Halloran BP, Van der Peet DL, Mulder CJ, et al: Comparison of MR enteroclysis with video capsule endoscopy in the investigation of small-intestinal disease. Abdom Imaging 2013;38:42-51. 